\title{
Classification of renal cell carcinoma based on expression of VEGF and VEGF receptors in both tumor cells and endothelial cells
}

\author{
Harriet M Kluger ${ }^{1}$, Summar F Siddiqui ${ }^{2}$, Cesar Angeletti ${ }^{2}$, Mario Sznol ${ }^{1}$, William K Kelly ${ }^{1}$, Annette M Molinaro ${ }^{3}$ \\ and Robert L Camp ${ }^{2}$
}

Recent development of antiangiogenic therapy for renal cell carcinoma (RCC) has significantly improved the treatment of these often refractory tumors. However, not all patients respond to therapy and assays for predicting outcome are needed. As a first step, we analyzed a retrospective cohort of tumors and assessed the ability of VEGF and VEGF receptors (VEGF-R1, -R2 and -R3) to classify tumors. We analyzed tissue microarrays containing 330 RCCs using a novel method of automated quantitative analysis of VEGF and VEGF-R expression by fluorescent immunohistochemistry. Expression of markers was separately quantified within three tissue components: tumor cells, endothelial cells and adjacent normal epithelium. VEGF and VEGF receptors were tightly coexpressed both within tumors and within adjacent normal cells (all $P$-values $<0.001$ ). Tumor cell expression of VEGF-R1 and -R2 was strongly and inversely correlated with vessel area $(P<0.0001)$. Unsupervised hierarchical clustering classified tumors by coordinated expression of VEGF and VEGF-Rs. The distribution of clear cell and papillary tumors was not significantly different between clusters. Clusters with high expression of VEGF and VEGF-Rs in the tumor cells exhibited poor survival when compared with the other clusters on uni- and multivariable analysis. VEGF and VEGF receptors exhibit a complex pattern of coordinated expression in RCC. Clustering tumors by VEGF and VEGF-R in tissue components demonstrates distinct tumor phenotypes with different outcomes, and may provide a means for determining which tumors will respond to what antiangiogenic therapies. Laboratory Investigation (2008) 88, 962-972; doi:10.1038/labinvest.2008.65; published online 14 July 2008

KEYWORDS: AQUA; biomarkers; prognosis; renal cell carcinoma; VEGF; VEGF receptors

Despite the recent success in treating advanced renal cell carcinoma (RCC) with antiangiogenic therapies, surprisingly little is known about expression of targets of these drugs in renal tumors and microvessels. There is some evidence to suggest that antiangiogenic agents target the microvasculature, ${ }^{1}$ but they may also target autocrine growth factor pathways within the tumor cells themselves. Two multitarget tyrosine kinase inhibitors have been approved by the Federal Drug Administration for use in unresectable RCC: sorafenib (Bayer Pharmaceuticals, Leverkusen, Germany and Onyx Pharmaceuticals, Emeryville, CA, USA) and sunitinib (Pfizer Inc., New York, NY, USA). Additional inhibitors of the VEGF pathway are in clinical trials. Sorafenib inhibits members of the RAF pathway, as well as VEGF-R2 (KDR/Flk-1), VEGF-R3 (Flt-4) and PDGFR- $\beta$, and sunitinib inhibits
VEGF-R2, PDGFR- $\beta$, Kit and Flt-3. Temsirolimus (Wyeth Pharmaceuticals, Madison, NJ, USA), an mTOR inhibitor, was also approved for advanced RCC and additional VEGF-R pathway-targeting agents are in clinical development.

The advent of these antiangiogenic agents into the clinic for RCC was preceded by advances in our understanding of tumor vasculature. Mutations in the von Hippel-Lindau (VHL) tumor suppressor gene have been found in approximately $75 \%$ of clear cell RCCs, ${ }^{2}$ resulting in induction of hypoxia-regulated genes in tumor cells, including VEGF. ${ }^{3-5}$ Additional steps are required for vessel formation, including loss of integrity of the extracellular matrix. ${ }^{6}$ Thus, inhibition of tumor angiogenesis is likely to require agents that target both the vessels and the malignant cells, as is evident

\footnotetext{
${ }^{1}$ Department of Medicine, Yale University School of Medicine, New Haven, CT, USA; ${ }^{2}$ Department of Pathology, Yale University School of Medicine, New Haven, CT, USA and ${ }^{3}$ Department of Biostatistics, Yale School of Public Health, New Haven, CT, USA

Correspondence: Dr RL Camp, MD, PhD, Department of Pathology, Yale University School of Medicine, 310 Cedar Street, PO Box 208023, New Haven, CT 06520, USA. E-mail: robert.camp@yale.edu

Received 12 March 2008; revised 23 May 2008; accepted 25 May 2008
} 
by the lack of efficacy of monotherapy that directly targets VEGF. ${ }^{1}$

Coupled with the clinical development of VEGF-Rtargeting therapies, there is an urgent need to develop biomarkers that predict response to these agents, as clearly only a subset of patients derive benefit from the drugs. Increased clinical benefit has been shown in patients who harbor VHL mutations, although other patients also derive benefit. $^{2}$ Additional approaches have been attempted to identify predictors of response, including assessment of target expression in tumor cells and tumor vasculature, interstitial fluid pressure, tumor oxygen tension, blood circulating endothelial cells, serum protein levels (such as VEGF) and imaging strategies that measure blood flow, as summarized by Jain $e t$ al. ${ }^{1}$

Immunohistochemistry (IHC)-based analysis of tumors is a practical approach to take for biomarker identification and validation, as IHC can be performed on small amounts of paraffin-embedded tissue. The typical first step in biomarker development is characterization of the biomarker in the disease population. Several studies have assessed expression of VEGF and VEGF receptors in RCC tumor cells; however few have separately analyzed the endothelial cell component, and none has used quantitative IHC to compare VEGF/ VEGF-R within these different tumoral elements. Mertz et $a l^{7}$ have used automated analysis to assess the microvessel density of RCC and found associations with aggressive disease, but did not look at the VEGF/VEGF-R pathway. Tsuchiya et $a l^{8}$ assessed expression of VEGF, VEGF-R1 (Flt-1) and VEGF-R2 in RCC tumor, adjacent normal renal tissue and endothelial cells by standard IHC and RT-PCR. However, their analyses only included 23 cases, and no association was made with clinical/pathological variables. Other smaller studies assessing VEGF/VEGF-Rs and microvessel density have been conducted. ${ }^{9-11}$ Jacobsen et al $^{12}$ assessed a relatively large cohort tissue microarray (TMA) for VEGF expression, but no assessment of VEGF-R expression and microvessel density was made. Our purpose was to assess expression of VEGF, VEGF-R1, VEGF-R2 and VEGF-R3 in three tissue components: RCC cells, endothelial cells and adjacent normal renal tissue on a large patient cohort with associated clinical/ pathological data. We also assessed vessel area (VA) in the tumors. To obtain more accurate, objective measures of expression, we used our new method of automated quantitative analysis (AQUA). This method has been validated, and can be more accurate than pathologist-based scoring of brown stain. ${ }^{13,14}$ As with some targeted therapies, it is possible that response to VEGF- or VEGF-R-targeting drugs might be associated with expression levels of targets in tumors or stroma, and quantitative assays need to be developed to predict response. Other markers that have both prognostic and predictive value have significantly impacted our ability to appropriately select therapeutic regimens for other cancers, and similar assays might be beneficial in selection of RCC patients for antiangiogenic therapies.

\section{MATERIALS AND METHODS}

\section{Tissue Microarray Construction}

TMAs were constructed as described. ${ }^{14}$ RCC cores from 334 patients, 294 with matching adjacent normal renal tissue, each measuring $0.6 \mathrm{~mm}$ in diameter, were spaced $0.8 \mathrm{~mm}$ apart on slides. Tumors were represented by two cores from different areas of tumor and adjacent normal kidney by one core. Specimens and clinical information were collected with the approval of a Yale University institutional review board. Histological subtypes included clear cell $(71 \%)$, papillary $(14 \%)$, chromophobe $(2 \%)$, mixed histology $(4 \%)$, oncocytomas $(6 \%)$ and sarcomatoid tumors $(3 \%)$. Oncocytomas were excluded from survival analyses. Among them, $8 \%$ had stage II and stage IV disease, $56 \%$ stage I and $28 \%$ stage IV; 12 were Fuhrman nuclear grade I, 52\% grade II, 27\% grade III and 9\% grade IV. See Supplementary Figure 1 for survival based on tumor type, stage and grade. Specimens were resected between 1987 and 1999, with follow-up of 2-240 months (median, 89.7). Age at diagnosis was $25-87$ years (median, 63). Treatment history was not available for the cohort.

\section{Immunohistochemistry}

For analysis of markers in tumor cells and normal epithelium, TMAs were stained with a cocktail of anti-cytokeratin and streptavidin (which binds endogenous biotin) and visualized with a green fluorophore (Alexa 488), in conjunction with an antibody to the target marker (VEGF or VEGF-R1, -R2 or -R3). Target markers were visualized by incubating with an appropriate horseradish peroxidase-conjugated secondary antibody and Cyanine- 5 tyramide. Cyanine- 5 was used because its far-red emission spectrum is outside of the range of tissue autofluorescence. For analysis of markers in microvessels, TMAs were stained with CD34 in conjunction with an antibody to the target marker. Details of methods and antibodies appear in the Supplementary Materials.

\section{Automated Image Acquisition and Analysis}

Images were acquired and analyzed using algorithms that have extensively been described. ${ }^{13}$ Briefly, monochromatic, high-resolution $(1280 \times 1024$ pixel $)$ images were obtained of each histospot. Tumor was distinguished from stroma by the cytokeratin/streptavidin signal, and endothelial cells were distinguished using CD34. The target signal (VEGF, VEGF$\mathrm{R} 1,-\mathrm{R} 2$ and -R3) from the tumor cells, adjacent normal epithelium or endothelial cells was scored on a scale of $0-255$, and expressed as the average signal intensity within the assayed component (AQUA score). Histospots containing $<3 \%$ tumor were excluded from further analysis. VA was calculated as the percent area of CD34 mask within a histospot.

\section{Statistical Analysis}

The StatView (SAS Institute Inc., Cary, NC, USA) and $\mathrm{R}^{15}$ software packages were used. AQUA scores for replicate tumor cores were averaged. Unsupervised hierarchical 
clustering was performed using TreeView and Cluster software. ${ }^{16}$ For clustering, AQUA scores were converted into $z$-scores and analysis was limited to cases with scores for $>80 \%$ of markers. ${ }^{17}$ The prognostic significance of parameters was assessed for predictive value using the Cox proportional hazard model (PHM) with RCC-specific survival as an end point. Kaplan-Meier survival curves were generated for patient subsets defined by the cluster analyses, with significance evaluated using the Mantel-Cox log-rank test and multivariate Cox PHMs. Correlations between markers were assessed using the Spearman's $\rho$-test. Variables were univariately and bivariately entered into Cox PHMs to assess the significance at $\alpha=0.05$. To evaluate whether individual variables and combinations of variables are able to
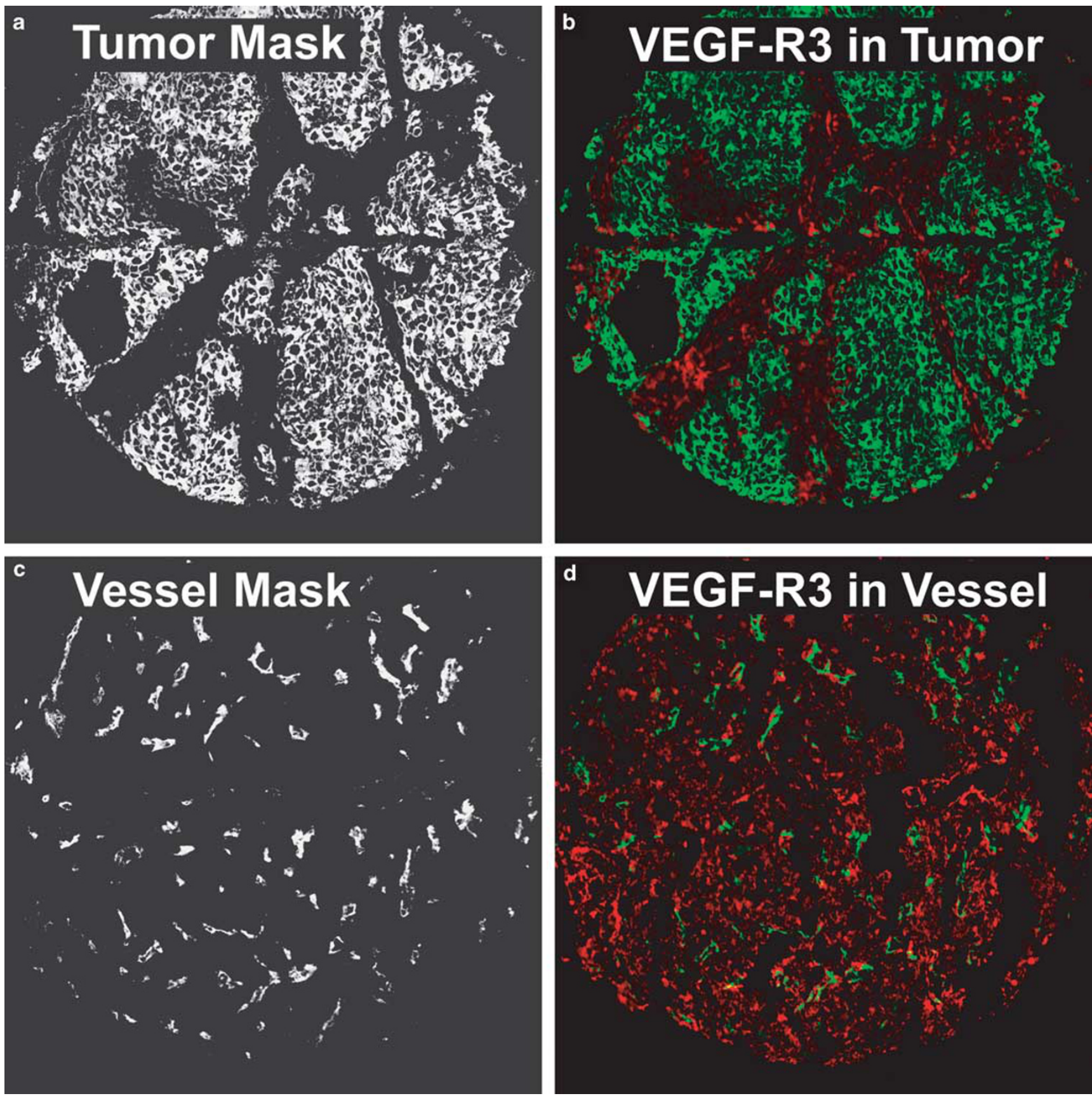

Figure 1 Localization and quantitation of VEGF-R3 in different tumor elements of an RCC histospot. (a) A cocktail of anticytokeratin and streptavidin was used to create a tumor cell mask. (b) VEGF-R3 expression in the tumor mask (green pixels) was distinguished from expression from other elements in the stroma (red pixels) using AQUA. AQUA then quantitates the VEGF-R3 expression in the tumor by summing the total intensity of VEGF-R3 expression in the mask divided by the number of masked pixels (ie, the sum of green pixel intensity/the number of green pixels). (c) An antibody against CD34 was used to create a tumor vessel mask. (d) VEGF-R3 expression in the vessels (green) is then independently assessed using the method in panel b. 
predict if a patient died of disease or was alive at 10 years, leave-one-out cross-validation (LOOCV) was employed with logistic regression models. This form of cross-validation iteratively splits the $N$ observations into a training set of size $\mathrm{N}-1$ and a test set of size 1 . At each iteration the coefficients for the logistic model are estimated based on $N-1$ observations in the training set, and used to predict the one observation in the test set. Misclassification was assessed based on whether the observation is incorrectly or correctly predicted. The model's prediction performance, or error, was calculated as the average misclassification over the $N$ iterations. The lower the average misclassification, the better the prediction. ${ }^{18}$ Confidence intervals (CIs) for prediction error estimates were constructed by nonparametric bootstrap resampling. ${ }^{19}$ See Supplementary Information for details.

\section{RESULTS}

VEGF and VEGF-R staining was localized to the cell membrane and cytoplasm of tumor cells, normal tubules and endothelial cells, with no significant nuclear staining. An
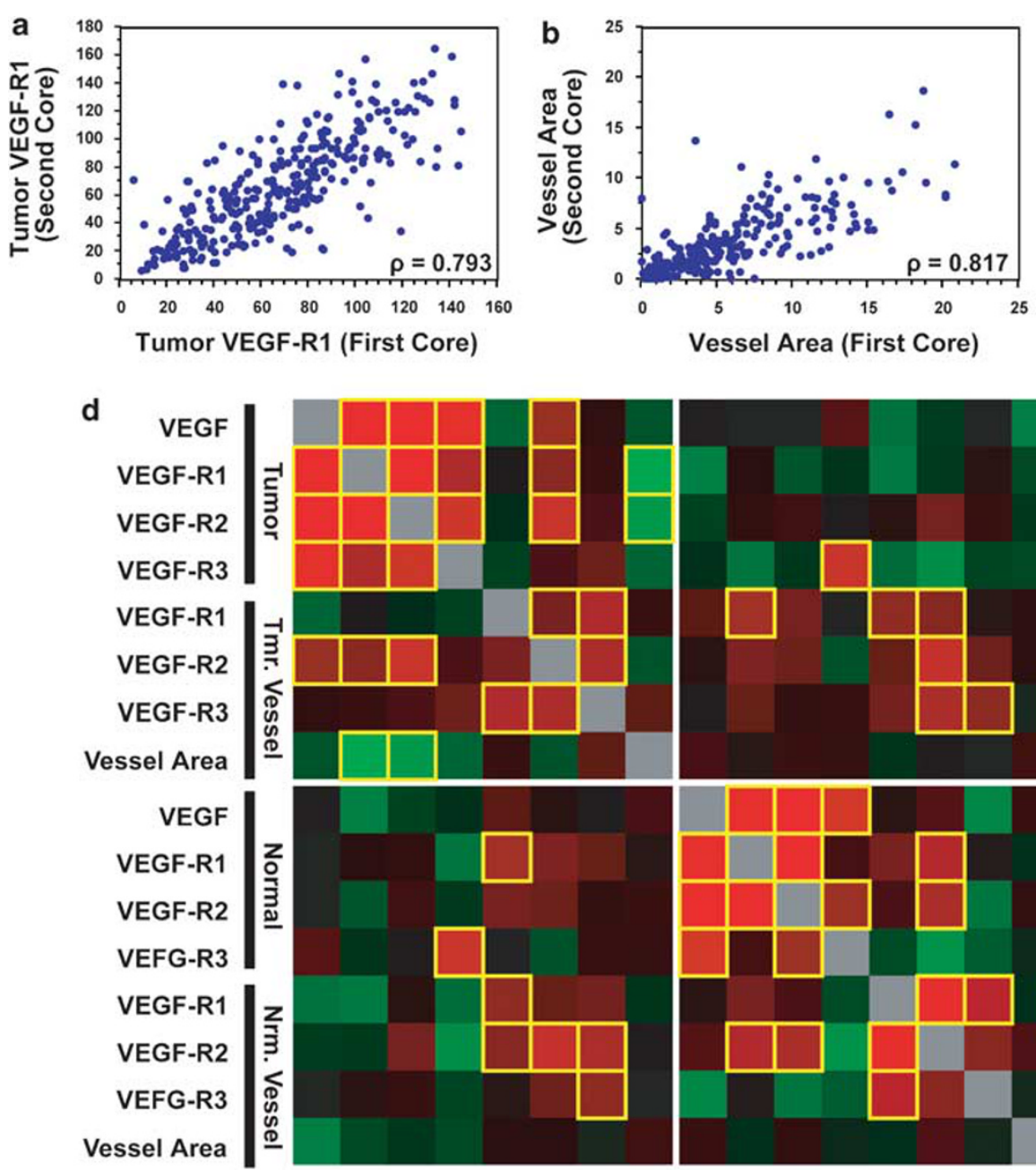
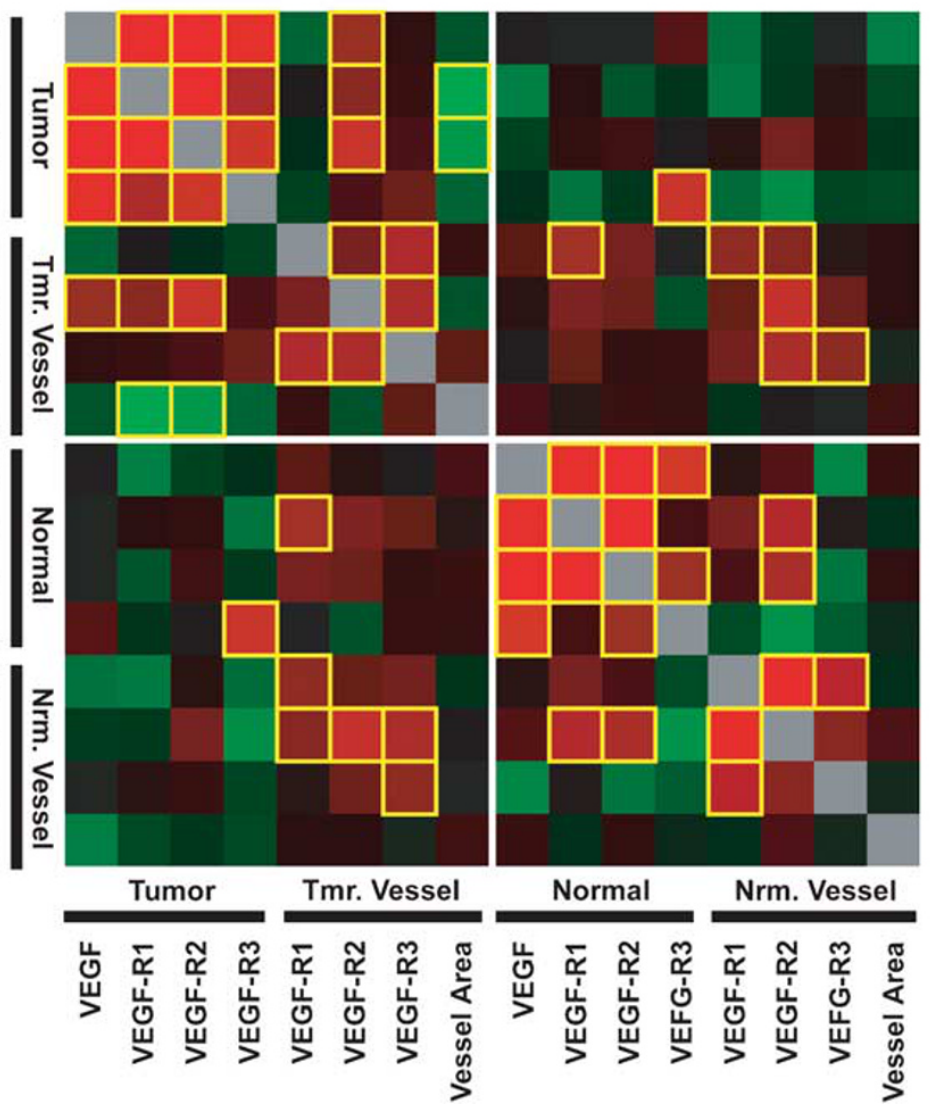

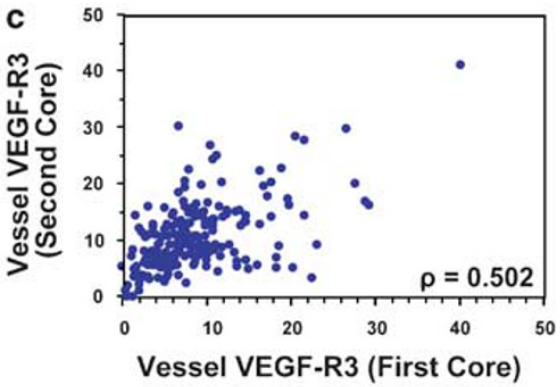

Spearman's $\rho$

$$
\begin{array}{r}
>0.50 \\
>0.25 \\
0 \\
<-0.25 \\
<-0.50
\end{array}
$$

p-value $<0.0004$

Figure 2 Correlations among VEGF, VEGF receptors and microvessel density. (a) Duplicate histocores taken for separate areas within 300 RCCs were analyzed for VEGF-R1 expression within the tumor mask. AQUA scores for VEGF-R1 expression for the first vs the second histocore are shown, and associations are expressed using Spearman's $\rho$. (b) Similarly, vessel area, expressed as the area covered by CD34 within the tumor demonstrates strong coreto-core correlation. (c) Expression of VEGF-R3 within the vessel area of matched histocores also shows strong correlation. (d) Spearman's $\rho$ correlation coefficients were determined for all combinations of VEGF, receptors and microvessel density. Direct correlations are shown in red, indirect correlations are in green. The intensity of color in each square represents the strength of the association. Correlations with a $P$-value of less than 0.0004 (corresponding to the Bonferroni correction for 112 multiple tests at an $\alpha$ of 0.05 ) are highlighted in yellow. Note that for ease of reading scores are represented twice. 
Table 1 VEGF and VEGF receptor expression in RCC

\begin{tabular}{|c|c|c|c|c|c|c|c|c|}
\hline & \multicolumn{2}{|c|}{ AQUA score } & \multicolumn{3}{|c|}{ Nuclear grade } & \multicolumn{3}{|c|}{ Stage } \\
\hline & Mean & s.d. & $1-2$ & $3-4$ & $P$-value & $|-| \mid$ & III-IV & $P$-value \\
\hline \multicolumn{9}{|l|}{ Tumor } \\
\hline VEGF & 36.9 & 23.6 & 34.8 & 41.0 & 0.0260 & 36.4 & 38.1 & 0.5608 \\
\hline VEGF-R1 & 43.8 & 21.0 & 40.3 & 50.5 & $<0.0001$ & 42.7 & 56 & 0.1658 \\
\hline VEGF-R3 & 32.7 & 12.0 & 31.5 & 35.0 & 0.0094 & 31.9 & 34.3 & 0.0883 \\
\hline \multicolumn{9}{|l|}{ Tumor vessel } \\
\hline VEGF-R1 & 10.3 & 5.8 & 9.9 & 11.2 & 0.0538 & 10.0 & 10.9 & 0.1854 \\
\hline VEGF-R2 & 21.6 & 6.2 & 22.2 & 21.3 & 0.2863 & 21.7 & 21.6 & 0.9368 \\
\hline \multicolumn{9}{|l|}{ Normal } \\
\hline VEGF & 36.2 & 17.8 & 37.5 & 33.0 & 0.0478 & 37.4 & 33.5 & 0.0921 \\
\hline VEGF-R1 & 33.4 & 13.9 & 34.1 & 32.0 & 0.2320 & 33.5 & 33.1 & 0.8014 \\
\hline VEGF-R2 & 61.3 & 19.8 & 62.2 & 59.9 & 0.3637 & 63.3 & 57.2 & 0.0128 \\
\hline VEGF-R3 & 27.1 & 10.6 & 27.1 & 27.3 & 0.9091 & 27.1 & 27.0 & 0.9153 \\
\hline \multicolumn{9}{|l|}{ Normal vessel } \\
\hline VEGF-R1 & 8.8 & 7.9 & 8.9 & 8.6 & 0.7386 & 9.0 & 8.3 & 0.4838 \\
\hline VEGF-R2 & 16.3 & 5.4 & 16.5 & 16.1 & 0.6045 & 16.4 & 16.2 & 0.8102 \\
\hline VEGF-R3 & 9.0 & 4.8 & 9.0 & 9.0 & 0.9783 & 8.5 & 9.9 & 0.0278 \\
\hline Vessel area & 5.5 & 2.9 & 5.3 & 5.6 & 0.4078 & 5.8 & 5.0 & 0.0307 \\
\hline
\end{tabular}

AQUA, automated quantitative analysis; VEGF, vascular endothelial growth factor.

Associations with $P<0.05$ are bolded.

Table 2 Multivariate regression of tumor vessel area vs VEGF/VEGF-R expression ${ }^{\mathrm{a}}$

\begin{tabular}{lrrrr}
\hline Marker & Coefficient & s.e. & $t$-value & $P$-value \\
\hline Intercept & 5.672 & 1.078 & 5.263 & $<0.0001$ \\
$\begin{array}{l}\text { Tumor } \\
\text { VEGF }\end{array}$ & 0.024 & 0.016 & 1.505 & 0.1338 \\
VEGF-R1 & -0.031 & 0.014 & -2.201 & 0.0288 \\
VEGF-R2 & -0.029 & 0.012 & -2.404 & 0.0170 \\
VEGF-R3 & -0.006 & 0.024 & -0.244 & 0.8074 \\
Vessel & & & & \\
VEGF-R1 & 0.066 & 0.061 & 1.093 & 0.2757 \\
VEGF-R2 & -0.034 & 0.043 & -0.786 & 0.4329 \\
VEGF-R3 & 0.071 & 0.035 & 2.052 & 0.0413 \\
\hline
\end{tabular}

${ }^{\mathrm{a}} P$-value for the model $=0.0018$. example of VEGF-R3 staining within endothelial and tumor cells is shown in Figure 1.

To account for intratumor heterogeneity, two separate sets of slides, each containing a core from a different area of the tumor for each patient, were used to evaluate expression of each marker. We assessed the correlation between the two cores for each marker and for VA using the Spearman's $\rho$-test. All scores from the two cores for the different markers within the tissue compartments correlated well $(P<0.0001)$, and examples are given in Figure 2a-c. Scores from corresponding cores were averaged to obtain a single concatenated score for each marker. The mean AQUA scores and the significance of the association with nuclear grade and stage by unpaired $t$-tests are shown in Table 1. Most notably, high VEGF/ VEGF-R expression in tumor cells was associated with high nuclear grade, as were high VEGF-R3 in vessels and VA. High VEGF-R2 in tumor and normal kidney, high VEGF-R3 in vessels and low VA were associated with stage III/IV disease. VEGF-R levels were higher on tumor cells than normal 
epithelium, and higher on tumor vessels than normal vessels (see Supplementary Table 2 for paired $t$-test $P$-values).

We assessed associations between markers by Spearman's $\rho$-test, as demonstrated in Figure 2d. Red squares represent positive correlations, and green represent anticorrelations. Squares with yellow perimeters represent strong associations between markers $(P<0.0004)$. We found strong coordinated expression of VEGF and VEGF-Rs within each tissue compartment, and a strong anticorrelation between VEGF-R1 and -R2 in tumor cells and VA.

Table 2 shows a multivariate regression model of the association between expression of VEGF and VEGF receptors on tumor and endothelial cells and VA. Expression of VEGF-R1 and -R2 on tumor cells was strongly and independently anticorrelated with VA, whereas high expression of VEGF-R3 on endothelial cells was strongly and independently correlated with VA.

By Cox univariate analysis of raw AQUA scores, we assessed the associations between markers and RCC-specific survival, as shown in Table 3. Markers associated with decreased survival include high stage, high nuclear grade, high tumor VEGF, VEGF-R1, -R2 and -R3, high VEGF-R3 in vessels, low tumor VA and low VEGF in adjacent normal kidney.

To assess the ability of each marker to predict patient outcome, we used LOOCV to calculate misclassification rates. Patients were dichotomized as either died of RCC at 10 years or alive at 10 years. Misclassification rates for each of the markers ranged from 0.24 to 0.27 , comparable to stage or grade (Table 3 ). However, none of the markers, or stage or grade, produced misclassification rates that were below the $95 \%$ CI of the null model. Note that in many cases, analyzing the ratio of tumoral expression to normal epithelial expression improved the misclassification rate.

We performed unsupervised hierarchical clustering of tumors by expression of VEGF/VEGF-Rs in tumor cells, microvessels and adjacent kidney. Markers clustered into three groups: those from tumor cells (red), normal epithelium (green), and microvessels (blue) (Figure 3a). Microvessels clustered together regardless of their original location (tumor or adjacent normal kidney). Tumors were classified into six distinct clusters: (1) low expression of all markers in tumor, normal kidney and microvessels (blue); (2) high in normal and low in tumor (green); (3) singularly high levels of VEGFR3 in tumor and normal cells (pink); (4) high tumor and microvessel expression (orange); (5) high in tumor only (red) and (6) unclassified (black). We then determined if clusters had significant differences in VA. Clusters with low VEGF/ VEGF-R tumor expression (blue and green) were associated with higher VA (Figure 3b). There was no clear association between cluster assignment and pathological subtype (Figure 3c). We note, for example, that $28 \%$ of clear cell cases were assigned to the blue cluster, compared to $20.5 \%$ of the papillary cases, but the number of cases in all nonclear cell subtypes was small, and definitive conclusions cannot be
Table 3 Continuous univariate analysis and 10 year dead of disease misclassification rates

\begin{tabular}{|c|c|c|c|c|}
\hline Marker & Hazard ratio & s.e. & $P$-value & $\begin{array}{c}\text { Misclassification } \\
\quad(95 \% \mathrm{Cl})\end{array}$ \\
\hline \multicolumn{5}{|l|}{ Stage } \\
\hline 34 vs 12 & 7.82 & 0.27 & 0.00 & $0.24(0.19-0.44)$ \\
\hline \multicolumn{5}{|l|}{ Nuclear grade } \\
\hline 34 vs 12 & 5.24 & 0.25 & 0.00 & $0.26(0.20-0.46)$ \\
\hline \multicolumn{5}{|l|}{ VEGF } \\
\hline Tumor & 1.51 & 0.14 & 0.00 & $0.26(0.21-0.32)$ \\
\hline Normal & 0.70 & 0.14 & 0.01 & $0.24(0.19-0.31)$ \\
\hline Difference & 1.50 & 0.10 & 0.00 & $0.24(0.18-0.29)$ \\
\hline \multicolumn{5}{|l|}{ R1 } \\
\hline Tumor & 1.62 & 0.17 & 0.01 & $0.26(0.22-0.32)$ \\
\hline Normal & 0.94 & 0.22 & 0.77 & $0.24(0.19-0.29)$ \\
\hline Difference & 1.39 & 0.14 & 0.02 & $0.24(0.19-0.30)$ \\
\hline \multicolumn{5}{|l|}{$R 2$} \\
\hline Tumor & 1.51 & 0.16 & 0.01 & $0.26(0.21-0.31)$ \\
\hline Normal & 0.64 & 0.23 & 0.05 & $0.24(0.18-0.30)$ \\
\hline Difference & 1.64 & 0.14 & 0.00 & $0.25(0.19-0.30)$ \\
\hline \multicolumn{5}{|l|}{ R3 } \\
\hline Tumor & 1.64 & 0.22 & 0.02 & $0.26(0.21-0.31)$ \\
\hline Normal & 0.90 & 0.21 & 0.62 & $0.24(0.20-0.30)$ \\
\hline Difference & 1.53 & 0.19 & 0.03 & $0.24(0.19-0.30)$ \\
\hline \multicolumn{5}{|l|}{$R 1$} \\
\hline Tumor vessel & 1.13 & 0.17 & 0.46 & $0.26(0.21-0.31)$ \\
\hline Normal vessel & 1.00 & 0.15 & 1.00 & $0.24(0.19-0.30)$ \\
\hline Difference & 1.05 & 0.14 & 0.71 & $0.24(0.19-0.30)$ \\
\hline \multicolumn{5}{|l|}{$R 2$} \\
\hline Tumor vessel & 1.44 & 0.30 & 0.22 & $0.27(0.21-0.32)$ \\
\hline Normal vessel & 0.93 & 0.33 & 0.82 & $0.22(0.17-0.29)$ \\
\hline Difference & 1.38 & 0.30 & 0.28 & $0.24(0.17-0.31)$ \\
\hline \multicolumn{5}{|l|}{ R3 } \\
\hline Tumor vessel & 1.51 & 0.17 & 0.02 & $0.26(0.20-0.30)$ \\
\hline Normal vessel & 1.41 & 0.20 & 0.08 & $0.24(0.18-0.30)$ \\
\hline Difference & 1.16 & 0.18 & 0.43 & $0.24(0.19-0.30)$ \\
\hline Tumor vessel area & 0.87 & 0.06 & 0.04 & $0.26(0.20-0.31)$ \\
\hline Normal vessel area & 0.87 & 0.13 & 0.29 & $0.24(0.18-0.29)$ \\
\hline Null model & & & & $0.26(0.21-0.31)$ \\
\hline
\end{tabular}

$\mathrm{Cl}$, confidence interval; s.e., standard error of the hazard ratio. Hazard ratios with $P<0.05$ are bolded.

drawn. Two clusters (orange and red) with high tumor expression of VEGF/VEGF-Rs exhibited worse survival by Kaplan-Meier analysis $(P=0.0007$; Figure 3d). 

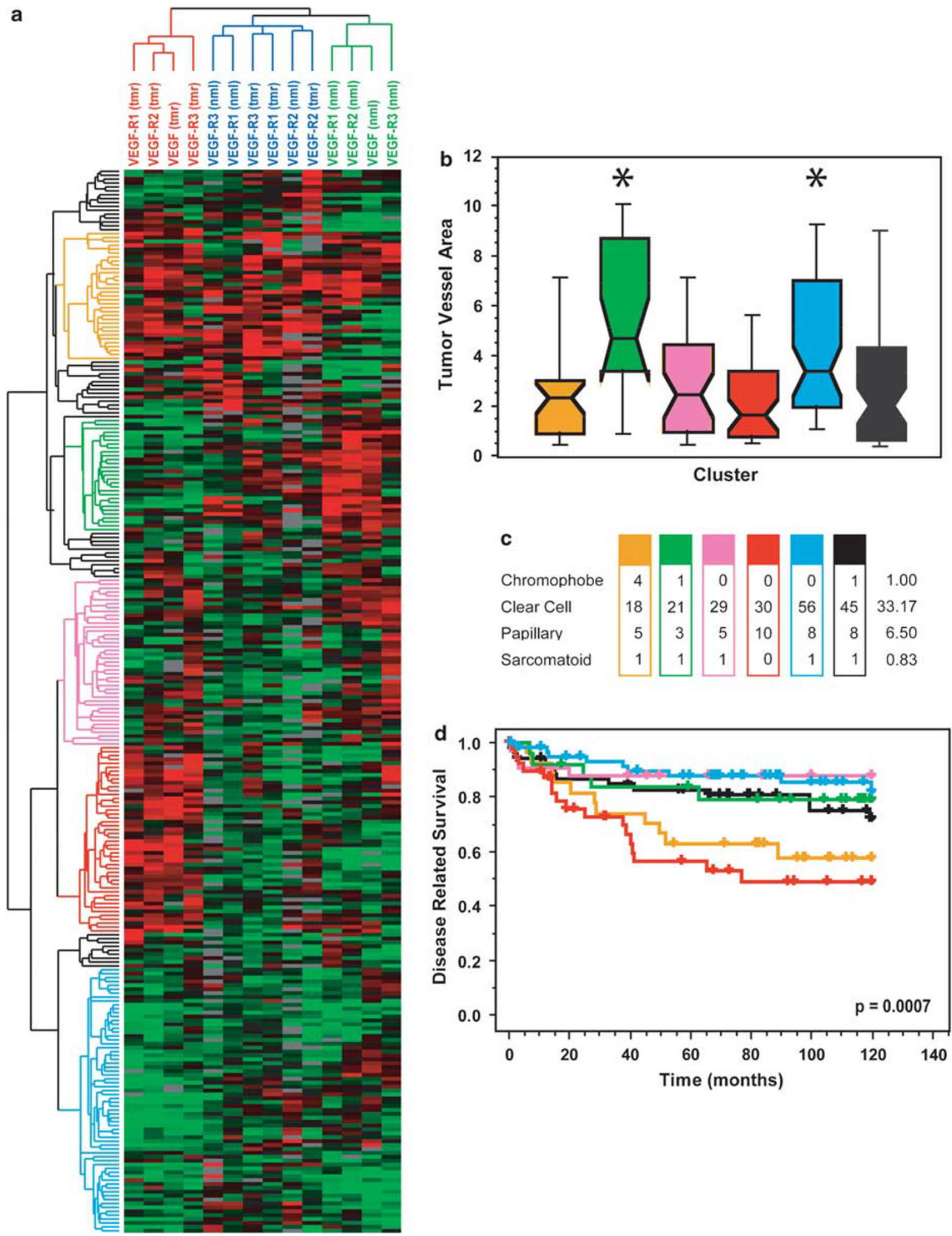

Figure 3 Unsupervised hierarchical clustering. (a) Clustering of RCCs based on their expression of VEGF and VEGF-Rs on tumor, vessel and adjacent normal tissue. Six distinct expression patterns were observed. (b) Tumor vessel areas of each cluster are shown. Lines on each bar represent the $95,75 \%$, mean, 25 and $5 \%$ intervals. Two clusters, denoted with an asterisk, have significantly higher vessel areas than the remaining clusters (both $P<0.0034$ by ANOVA). (c) With the exception of chromophobe carcinomas, the distributions of tumor subtypes are not significantly different between clusters. The expected number per cluster is denoted on the right. (d) Survival analysis of the identified clusters shows that two clusters-both with high VEGF/VEGFR tumor expression-have decreased survival relative to the remaining cases. Significance was determined using the log-rank test. 
Table 4 Multivariate analysis of 10-year disease-related survival

\begin{tabular}{|c|c|c|c|c|}
\hline Marker & $N$ & $P$ & $\begin{array}{l}\text { Relative } \\
\text { risk }\end{array}$ & $\begin{array}{l}\text { 95\% confidence } \\
\text { interval }\end{array}$ \\
\hline Cluster & & 0.0273 & & \\
\hline Low & 184 & & 1.00 & \\
\hline High & 74 & & 1.86 & $1.07-3.22$ \\
\hline Histology & & 0.5479 & & \\
\hline Clear cell & 198 & & 1.00 & \\
\hline Non & 60 & & 0.83 & $0.45-1.54$ \\
\hline Stage & & $<0.0001$ & & \\
\hline 1 & 151 & & 1.00 & \\
\hline 2 & 17 & 0.3396 & 0.39 & $0.05-2.95$ \\
\hline 3 & 77 & $<0.0001$ & 3.90 & $2.13-7.16$ \\
\hline 4 & 13 & $<0.0001$ & 9.47 & $4.15-21.63$ \\
\hline Nuclear grade & & 0.0004 & & \\
\hline 1 & 35 & & 1.00 & \\
\hline 2 & 131 & 0.8303 & 1.12 & $0.33-3.99$ \\
\hline 3 & 71 & 0.1547 & 2.49 & $0.71-8.72$ \\
\hline 4 & 21 & 0.0141 & 5.15 & $1.39-19.09$ \\
\hline
\end{tabular}

On multivariable analysis, assignment to a cluster with high VEGF/VEGF-R was an independent predictor of poor survival, as were nuclear grade and stage (Table 4). Cluster assignment of the clear cell RCC patient subset produced similar results by Kaplan-Meier analysis, and was similarly significant on multivariable analysis (Supplementary Figure 2).

Next we assessed whether the addition of one or more markers in the VEGF/VEGF-R family could improve the misclassification rate of stage and grade (Figure 4). The ratio of VEGF-R1 in tumor $v s$ normal, which lowered the misclassification rate from 26 to $18.3 \%$, had the largest impact of all markers (Figure 4a). As expression of VEGF/VEGF-Rs is tightly associated in tumors, we were interested in interactions between these molecules that might have significant positive or negative associations with survival. We noted two such interactions: (1) a positive interaction between VEGF and VEGF-R1, and (2) a negative interaction between VEGF$\mathrm{R} 2$ and VEGF-R1 (Figure $4 \mathrm{~b}$ and $\mathrm{c}$ ). The combination of these two interactions in a logistic multivariate model with stage and grade reduced the misclassification rate to $17.4 \%$ (Figure 4a).

\section{DISCUSSION}

We quantitatively assessed VA and expression of VEGF/ VEGF-Rs in tumor, endothelial cells and adjacent normal renal tissue on a large cohort of RCC patients, and evaluated the association between expression and RCC-specific survival. AQUA gives objective, continuous measures of expression, rather than arbitrary pathologist-based divisions of staining into ordinal scores, or 'high/low', and can separately assess expression in different tumor components (eg, microvessels). Expression of VEGF and VEGF receptors, particularly VEGF-R1 and VEGF-R2, was highly correlated within each tissue component. Expression patterns were very similar in endothelial cells within the tumor and within the adjacent normal kidney, presumably because the vessels originate from the same primary cells. No clear differences in expression patterns were seen based on histological subtypes. Expression of VEGF/VEGF-Rs in tumors was strongly associated with nuclear grade, and these markers individually were not independent predictors of survival.

Unsupervised hierarchical clustering of samples based on expression patterns of markers in the tissue components revealed distinct patient clusters with divergent outcome; most notably, patients with high VEGF and VEGF-Rs in tumor cells had worse outcome when compared to the other clusters. The expression of VEGF-Rs on tumor vessels did not affect outcome. This classification of patients retained its prognostic significance on multivariable analysis and improved the misclassification rates of models that contained nuclear grade and stage.

One potential disadvantage of TMAs as opposed to whole tissue specimens is the difficulty in assessing heterogeneity of tumors. Of particular concern is the possibility that different areas of tumors might have different VA. Here we demonstrate a remarkably strong correlation between VAs from the two histospots from the same patient. We also found strong intrapatient correlations for expression of all the markers studied.

The reason for the anticorrelation between expression of VEGF-R1 and -R2 in tumor cells and VA is unknown, but may involve a 'steal' effect where tumors with high VEGF-R expression bind VEGF before it can be secreted to promote neovascularization. VEGF-R3 on endothelial cells was positively correlated with VA, as expected. Smaller studies by others have shown variable associations between clinical outcome and microvessel density. ${ }^{10,20}$ Kurban et al $l^{6}$ showed that VHL mutations resulting in upregulation of HIF $1 \alpha$ are associated with decreased microvessel density and low invasive potential, consistent with our results.

Our findings of an association between high VEGF-R1 and -R2 levels in tumor cells and decreased survival are contrary to those of Ljungberg et al, ${ }^{11}$ who assessed mRNA levels of these receptors in 104 patients. The difference might be related to sample size, inconsistencies between mRNA and protein levels and inclusion of stromal elements when performing RT-PCR on snap-frozen specimens.

Staging of RCC is important for determining prognosis and selecting high-risk patients for systemic therapies as they become available. For example, patients with stage III tumors have cure rates of $38-70 \%$ with nephrectomy alone, and 

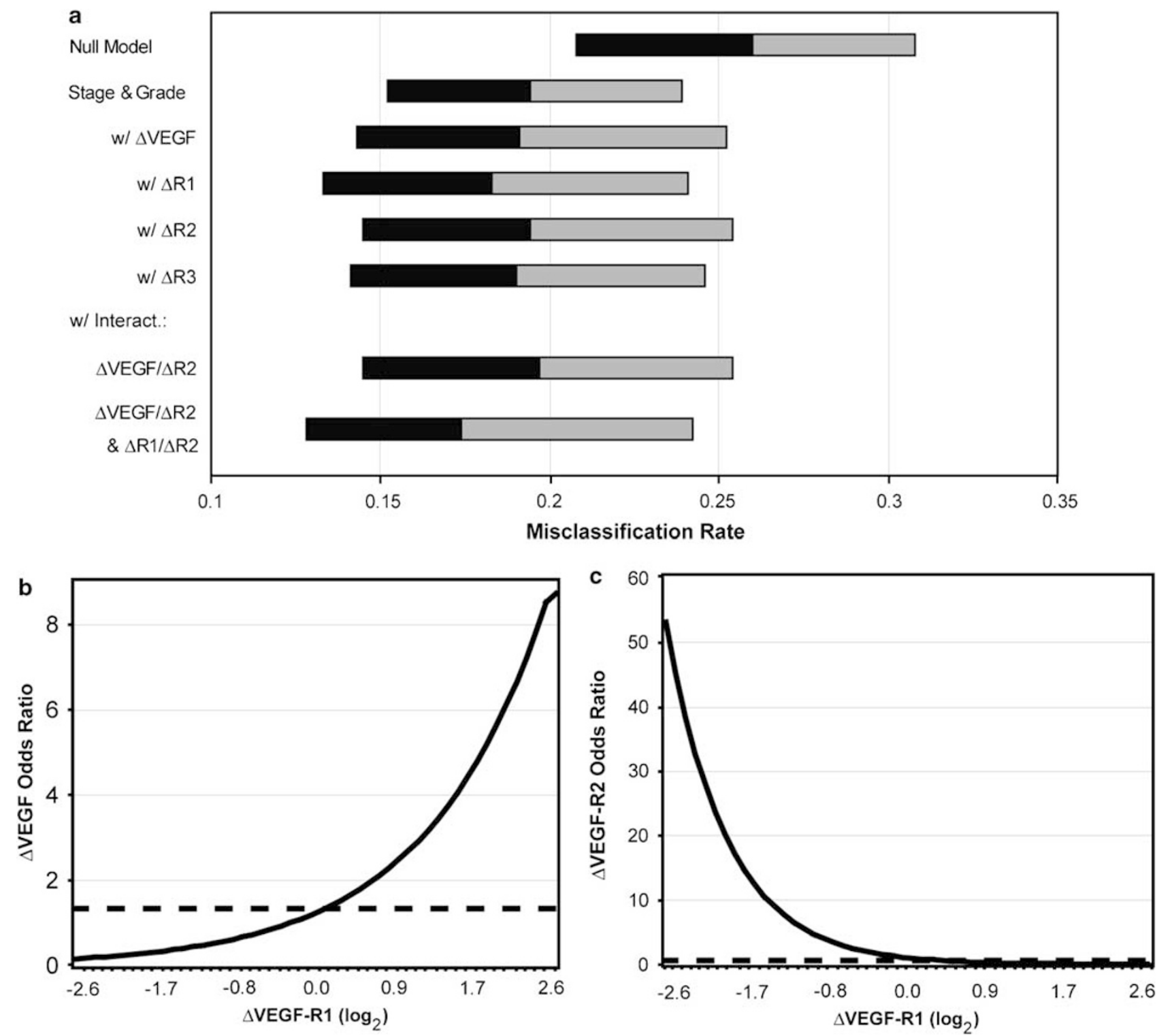

Figure 4 Misclassification rates of multivariate logistic models. All models were calculated using dead of disease at 10 years as a binary variable. Makers were assessed as a ratio of tumor expression to normal epithelial expression $(\Delta)$. All models included stage and grade. The ability of various models to predict outcome was assessed using LOOCV (a). 95\% confidence intervals are shown in black (lower) and gray (upper). Analysis of stage and grade improves the misclassification of outcome from 26 (using a null model) to $19.6 \%$. Adding VEGF or VEGF-Rs to this model does not significantly improve the misclassification rate. Two interesting interactions were noted: (b) shows that the ability of VEGF to predict outcome (y axis) improves on tumors with higher levels of VEGF-R1 ( $x$ axis). Without taking VEGF-R1 into account, the odds ratio for VEGF is 1.49 (dashed line). In contrast, (c) shows the ability of VEGF-R2 to predict outcome improves on tumors with lower VEGF-R1 levels. The dashed line shows the odds ratio of VEGF-R2 without the R1 interaction (0.71). The addition of the interactions outlined in panels $b$ and $c$ improves the misclassification rate to $17.4 \%$ (a).

adjuvant therapies are being investigated to increase cure rates. $^{21}$ Ideally these investigations should focus on patients with the worst prognosis. At present there are no approved adjuvant therapies for RCC, but a large phase III randomized trial is underway comparing sunitinib, sorafenib and placebo. Molecular markers in primary specimens highly associated with survival, such as profiles of high VEGF/VEGF-Rs, could improve staging for nonmetastatic patients, and enable us to identify high-risk patients more likely to benefit from adjuvant therapy, thus avoiding toxicity for patients who could be cured by resection alone. ${ }^{22}$

In addition to a potential role as a prognostic classifier, our findings have very important implications for therapeutic applications of VEGF and VEGF-R inhibitors in RCC. Metastatic RCC has been traditionally a dismal disease, due to resistance to chemotherapy. However, sunitinib and sorafenib have dramatically revolutionized the therapeutic approach to metastatic RCC. As with some other targeted 
therapies, there might be an association between target expression and response to therapy. It is yet unclear whether sunitinib and sorafenib inhibit RCC tumor growth by affecting tumor epithelial cells, endothelial cells or proangiogenic functions of adjacent normal kidney. In our retrospective cohort of clinical specimens there is variability in VEGF/VEGF-R expression, and expression patterns define distinct groups of patients with clear differences in prognosis. Our results show that high VEGF/VEGF-R in tumor cells is predominantly associated with poor survival. On the other hand, patients whose tumors have higher VA have improved survival, and lower levels of VEGF/VEGF-R in their tumor cells. Studies are needed to determine whether agents that target VEGF-R2 are more likely to be effective in patients whose tumor cells have higher target expression (and thus decreasing proliferation of tumor cells) or in patients whose tumors are more vascular and have lower levels of VEGF-Rs in tumor cells. Moreover, it is possible that small molecule inhibitors that target the receptors might be effective in a different subset of patients than those that will benefit from VEGF-targeting agents such as bevacuzumab. Given that sunitinib and sorafenib only benefit a subset of metastatic RCC patients, ${ }^{23,24}$ improved patient selection based on predictive biomarkers might improve the therapeutic ratio of these agents, as administration of these drugs is associated with some toxicity. Our studies have only addressed the expression of VEGF/VEGF-Rs on primary tumors. We are currently assessing whether metastatic tumor deposits express VEGF/VEGF-R levels at the same or different levels than the primary tumors from which they arose.

Some of the studies of sunitinib and sorafenib in RCC excluded nonclear cell subtypes. Our data show no clear differences in expression patterns of VEGF-Rs between the histological subtypes, suggesting that targeting these receptors might be effective in other RCC subtypes as well, as recently reported. ${ }^{25}$

In summary, our study shows that VEGF and VEGF receptors exhibit a complex pattern of coordinated expression in RCC. Although our observations were made in an untreated patient population, they may have significant therapeutic implications in the selection of antiangiogenic therapies for the tumors in different clusters. Clustering of tumors based on VEGF and VEGF receptors in tissue components may provide a prognostic assay and might also provide a means for determining which tumors will respond to what antiangiogenic therapies. Prospective studies are needed to confirm the prognostic role of VEGF and VEGF-R expression patterns, as well as the predictive value of expression patterns in patients treated with VEGF pathwaytargeting agents, preferably using quantitative methods of protein measurement. Future clinical trials incorporating these agents for RCC should stratify patients based on VEGF/VEGF-R expression, with the goal of developing an assay for improved patient selection for antiangiogenic agents.
Supplementary Information accompanies the paper on the Laboratory Investigation website (http://www.laboratoryinvestigation.org)

\section{ACKNOWLEDGEMENT}

Dr Kluger, Dr Molinaro and Dr Camp were supported by NIH Grants R0-1 CA115756-01, K-22 CA123146 and R21 CA116265, respectively.

\section{DISCLOSURE/DUALITY OF INTEREST}

Dr Camp is a stockholder and consultant to HistoRx, a private corporation to which Yale University has given exclusive rights to produce and distribute the software and technologies embedded in the AQUA technology.

1. Jain RK, Duda DG, Clark JW, et al. Lessons from phase III clinical trials on anti-VEGF therapy for cancer. Nat Clin Pract Oncol 2006;3:24-40.

2. Rini $\mathrm{Bl}$, Jaeger $\mathrm{E}$, Weinberg $\mathrm{V}$, et al. Clinical response to therapy targeted at vascular endothelial growth factor in metastatic renal cell carcinoma: impact of patient characteristics and von Hippel-Lindau gene status. BJU Int 2006:98:756-762.

3. Na X, Wu G, Ryan CK, et al. Overproduction of vascular endothelial growth factor related to von Hippel-Lindau tumor suppressor gene mutations and hypoxia-inducible factor-1 alpha expression in renal cell carcinomas. J Urol 2003;170(2 Part 1):588-592.

4. Takahashi A, Sasaki H, Kim SJ, et al. Markedly increased amounts of messenger RNAs for vascular endothelial growth factor and placenta growth factor in renal cell carcinoma associated with angiogenesis. Cancer Res 1994;54:4233-4237.

5. Igarashi $\mathrm{H}$, Esumi $M$, Ishida $\mathrm{H}$, et al. Vascular endothelial growth factor overexpression is correlated with von Hippel-Lindau tumor suppressor gene inactivation in patients with sporadic renal cell carcinoma. Cancer 2002;95:47-53.

6. Kurban G, Hudon V, Duplan E, et al. Characterization of a von Hippel Lindau pathway involved in extracellular matrix remodeling, cell invasion, and angiogenesis. Cancer Res 2006;66:1313-1319.

7. Mertz KD, Demichelis F, Kim R, et al. Automated immunofluorescence analysis defines microvessel area as a prognostic parameter in clear cell renal cell cancer. Hum Pathol 2007;38:1454-1462.

8. Tsuchiya N, Sato K, Akao T, et al. Quantitative analysis of gene expressions of vascular endothelial growth factor-related factors and their receptors in renal cell carcinoma. Tohoku J Exp Med 2001;195:101-113.

9. Yildiz E, Gokce $\mathrm{G}$, Kilicarslan $\mathrm{H}$, et al. Prognostic value of the expression of Ki-67, CD44 and vascular endothelial growth factor, and microvessel invasion, in renal cell carcinoma. BJU Int 2004;93:1087-1093.

10. Fukata $S$, Inoue K, Kamada $M$, et al. Levels of angiogenesis and expression of angiogenesis-related genes are prognostic for organspecific metastasis of renal cell carcinoma. Cancer 2005;103:931-942.

11. Ljungberg BJ, Jacobsen J, Rudolfsson $\mathrm{SH}$, et al. Different vascular endothelial growth factor (VEGF), VEGF-receptor 1 and -2 mRNA expression profiles between clear cell and papillary renal cell carcinoma. BJU Int 2006;98:661-667.

12. Jacobsen J, Grankvist K, Rasmuson T, et al. Expression of vascular endothelial growth factor protein in human renal cell carcinoma. BJU Int 2004;93:297-302.

13. Camp RL, Chung GG, Rimm DL. Automated subcellular localization and quantification of protein expression in tissue microarrays. Nat Med 2002;8:1323-1327.

14. Camp RL, Dolled-Filhart M, King BL, et al. Quantitative analysis of breast cancer tissue microarrays shows that both high and normal levels of HER2 expression are associated with poor outcome. Cancer Res 2003;63:1445-1448.

15. Ihaka R, Gentleman R. R: a language for data analysis and graphics. J Comp Graph Stat 1996;5:299-314.

16. Eisen MB, Spellman PT, Brown PO, et al. Cluster analysis and display of genome-wide expression patterns. Proc Natl Acad Sci USA 1998;95:14863-14868.

17. Boyd JC, Lacher DA. A multi-stage Gaussian transformation algorithm for clinical laboratory data. Clin Chem 1982;28:1735-1741.

18. Molinaro AM, Simon R, Pfeiffer RM. Prediction error estimation: a comparison of resampling methods. Bioinformatics 2005;21:3301-3307.

19. Efron B, Tibshirani R. An Introduction to the Bootstrap, Vol. Xvi Chapman \& Hall: New York, 1993. 
20. Nativ O, Sabo E, Reiss A, et al. Clinical significance of tumor angiogenesis in patients with localized renal cell carcinoma. Urology 1998;51:693-696.

21. Guinan PD, Vogelzang NJ, Fremgen AM, et al. Renal cell carcinoma: tumor size, stage and survival. Members of the Cancer Incidence and End Results Committee. J Urol 1995;153(3 Part 2): 901-903.

22. Guinan $\mathrm{P}$, Sobin LH, Algaba F, et al. TNM staging of renal cell carcinoma: workgroup no. 3. Union International Contre le Cancer (UICC) and the American Joint Committee on Cancer (AJCC). Cancer 1997;80:992-993.
23. Escudier BS, T E. Randomized phase III trial of the Raf kinase and VEGFR inhibitor sorafenib (BAY 43-9006) in patients with advanced renal cell carcinoma (RCC). J Clin Onc 2005; 23(16s):4510.

24. Motzer RJ, Hutson TE, Tomczak $P$, et al. Sunitinib versus interferon alfa in metastatic renal-cell carcinoma. N Engl J Med 2007;356: 115-124.

25. Plantade A, Rini B, Negrier S, et al. Treatment outcome for metastatic papillary and chromophobe renal cell carcinoma (RCC) patients treated with tyrosine-kinase inhibitors (TKIs) sunitinib and sorafenib. J Clin Onc 2007;25(18s): 5037. 